\title{
Dimethylfumarate inhibits CXCL10 via haem oxygenase-1 in airway smooth muscle
}

\author{
Petra Seidel*,\#, Katrin E. Hostettler*, J. Margaret Hughes\#, \\ Michael Tamm* and Michael Roth*
}

ABSTRACT: CXCL10 stimulates mast cell infiltration into airway smooth muscle bundles and, thus, activate cytokine secretion and airway smooth muscle cell (ASMC) proliferation. Dimethylfumarate (DMF) reduces cytokine secretion by lymphocytes and ASMC proliferation through haem oxygenase (HO)-1. Therefore, we investigated the potency of DMF to inhibit tumour necrosis factor (TNF)- $\alpha$ - and interferon (IFN)- $\gamma$-induced CXCL10 secretion by human ASMCs.

Human primary ASMCs were pre-incubated with DMF and/or fluticasone and/or glutathione ethylester before cells were stimulated with IFN- $\gamma$ and/or TNF- $\alpha$.

DMF inhibited CXCL10 secretion and increased HO-1 levels, and p38 mitogen-activated protein kinase (MAPK) inhibition reduced DMF-dependent HO-1 expression. The DMF effect on CXCL10 secretion was abrogated by pre-treatment with HO-1 small interfering RNA (siRNA). Glutathione supplementation reversed all DMF effects on CXCL10 secretion and p38 MAPK phosphorylation. Importantly, combining DMF with fluticasone further reduced CXCL10 secretion. In addition, DMF inhibited IFN- $\gamma$-induced CXCL10 secretion. This effect was compensated by glutathione supplementation or by pre-treatment with HO-1 siRNA. In addition, DMF reduced TNF- $\alpha$-induced granulocyte colony-stimulating factor (G-CSF) secretion but had no effect on INF- $\gamma$-induced GCSF secretion.

In human primary ASMCs, DMF inhibits CXCL10 secretion by reducing the cellular glutathione level and by activation of p38 MAPK and HO-1. Therefore, DMF may reduce airway inflammation in asthma by a glucocorticoid-independent pathway.

KEYWORDS: Airway smooth muscle, cell inflammation, dimethylfumarate, glutathione, haem oxygenase-1, interferon- $\gamma$, tumour necrosis factor- $\alpha$

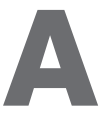

sthma is a chronic inflammatory disease of the airways that is characterised by airway hyperresponsiveness, increased bronchoconstriction and an increased airway wall thickness [1]. Airway inflammation in asthma involves tissue-forming cells, such as epithelial cells, fibroblasts and airway smooth muscle cells (ASMCs), as well as circulating immune cells, mainly mast cells, T-cells and eosinophils [2]. Activated ASMCs secrete a wide range of pro-inflammatory factors when stimulated by cytokines such as tumour necrosis factor (TNF)- $\alpha$, thereby recruiting more inflammatory cells to the inflamed area [3].

CXCL10 is a potent mast cell chemoattractant that contributes to the pathogenesis of asthma [4]. Support for a contribution of CXCL10 to asthma pathology comes from the observation that cell culture medium collected from activated asthmatic ASMCs induced chemotaxis of human lung mast cells [5]. This effect was reduced when CXCL10 was neutralised or when its receptor CXCR3 was blocked [6]. In addition, ASMCs of asthma patients secreted more CXCL10 than cells obtained from healthy controls [7]. This observation correlated with the increased number of active mast cells in airway smooth muscle bundles of asthma patients. Furthermore, CXCL10 secretion and mast cell number correlated with airway hyperresponsiveness [4] Inhaled glucocorticoids are the most frequently prescribed anti-inflammatory medications in asthma [7]. However, in $10 \%$ of asthma patients, airway inflammation is not well controlled by conventional therapies and these patients account for $>50 \%$ of the total asthma healthcare costs [8]. In cell culture models, it has been shown that the inflammatory interferon (IFN)- $\gamma$ signalling pathway up-regulated CXCL10 expression by ASMCs and that this was steroid resistant [9]. Therefore, new therapeutic options are needed to control airway inflammation in asthma.

Anecdotally, dimethylfumarate (DMF; also known as BG00012) has been reported to reduce asthma
AFFILIATIONS

*Pulmonary Cell Research, Dept of Biomedicine and Pneumology, Dept of Internal Medicine, University Hospital Basel, Basel, Switzerland. \#Faculty of Pharmacy, University of Sydney, Sydney, Australia.

CORRESPONDENCE

Michael Roth

University Hospital Basel

Pneumology

Petersgraben 4

CH-4031 Basel

Switzerland

E-mail: rothmic@uhbs.ch

Received:

April 212011

Accepted after revision:

April 042012

First published online:

July 122012 
symptoms and to improve the quality of life of psoriasis patients who also suffer from asthma. In vitro, DMF modified the metabolism of intracellular reduced glutathione $(\mathrm{GSH})$ and thereby reduced the expression of pro-inflammatory cytokines $[10,11]$. In human peripheral blood mononuclear cells, DMF depleted intracellular GSH and induced haem oxygenase (HO)-1, while it inhibited the secretion of interleukin (IL)-12 and IFN- $\gamma$. These effects were compromised when GSH was supplemented [10]. Regarding the beneficial effect of DMF in multiple sclerosis, it was supposed to act via the cytoprotective transcription factor Nrf-2, which is activated in the absence of GSH [9]. In human ASMCs, we showed that DMF inhibited TNF- $\alpha$-induced secretion of IL-6, eotaxin and RANTES (regulated on activation, T-cell expressed and secreted) [12]. Furthermore, we observed an inhibitory effect of DMF through HO-1 on platelet-derived growth factor (PDGF)-BB-induced ASMC proliferation [13]. These beneficial effects of DMF together with its clinical long-term safety profile make it an interesting drug for the therapy of chronic inflammatory lung diseases [14].

In this study, we investigated the effect of DMF on $\mathrm{HO}-1$ expression and its role on TNF- $\alpha$-and/or IFN- $\gamma$-induced CXCL10 and granulocyte colony-stimulating factor (G-CSF) secretion by human ASMCs. Furthermore, we studied the role of mitogen-activated protein kinase (MAPK) activation in the context of DMF-stimulated HO-1 expression. We also assessed
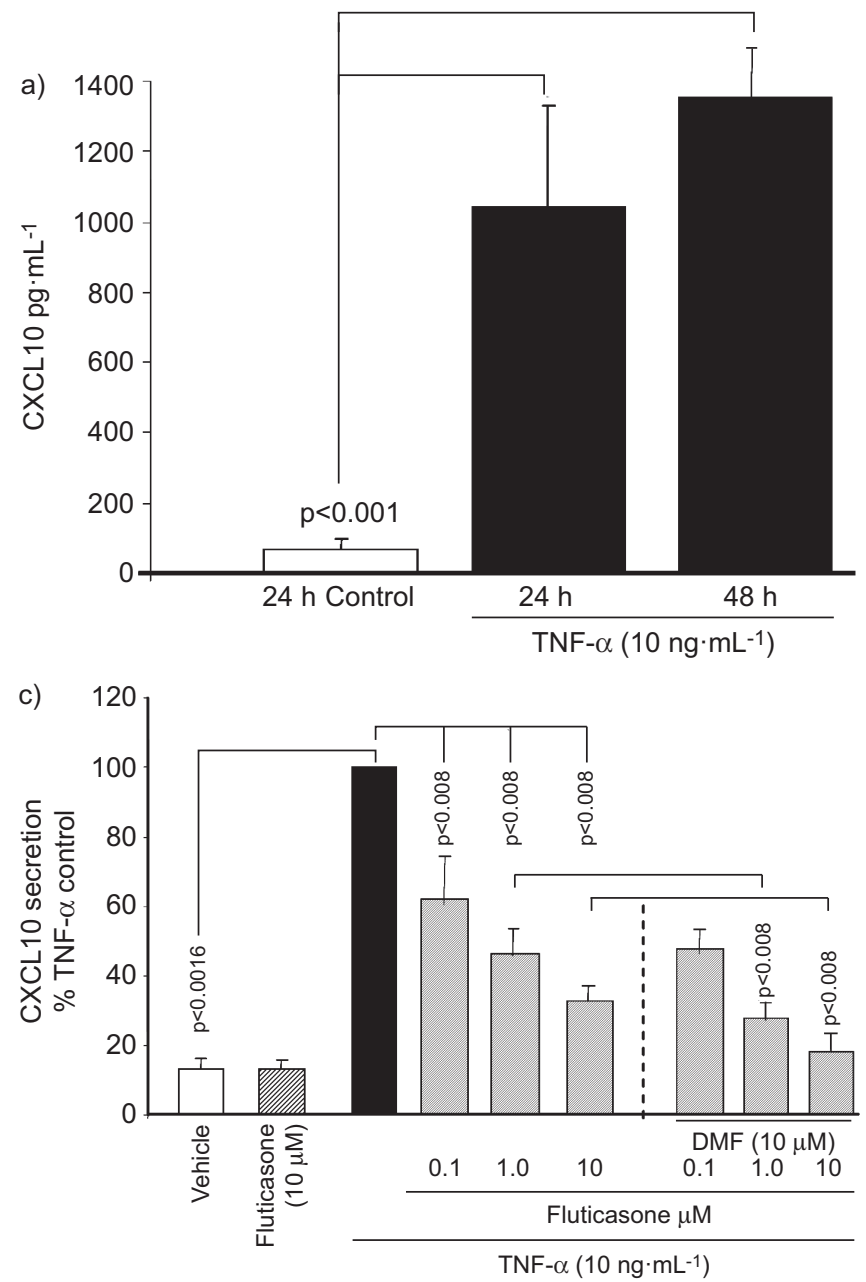

the effect of GSH supplementation on this signalling system together with possible steroid-saving properties of DMF.

\section{MATERIAL AND METHODS Lung tissue specimens}

All specimens used in this study were obtained from the Dept of Internal Medicine, University Hospital Basel (Basel, Switzerland) with the approval of the local ethical committees and the written consent of all patients.

\section{Isolation and culture of human ASMCs}

Human primary ASMCs were isolated and grown from bronchi of healthy lung tissue obtained from unused lung transplants and were established as previously described [15]. ASMCs were grown in RPMI-1640 (ThermoTrace, Melbourne, Australia) supplemented with $5 \%(\mathrm{v} / \mathrm{v})$ heat-inactivated fetal bovine serum (FBS), $1 \times$ Minimal Essential Medium vitamin mix, $100 \mathrm{U} \cdot \mathrm{L}^{-1}$ penicillin, $100 \mu \mathrm{g} \cdot \mathrm{mL}^{-1}$ streptomycin, $0.25 \mu \mathrm{g} \cdot \mathrm{mL}^{-1}$ amphotericin B (all GIBCO/BRL, Melbourne, Australia), $25 \mathrm{mM}$ hydroxyethyl piperazine ethane sulfonic acid and $2 \mathrm{mM}$ L-glutamine (both ThermoTrace) in a humidified 5\% carbon dioxide atmosphere at $37^{\circ} \mathrm{C}$. ASMCs were used between passages 5 and 8 .

\section{Drug preparation}

All chemicals were obtained from Sigma (Buchs, Switzerland). DMF (0.1-100 $\mu \mathrm{M})$, SB203580 (10 $\mu \mathrm{M})$, fluticasone $(0.1-10 \mu \mathrm{M})$,

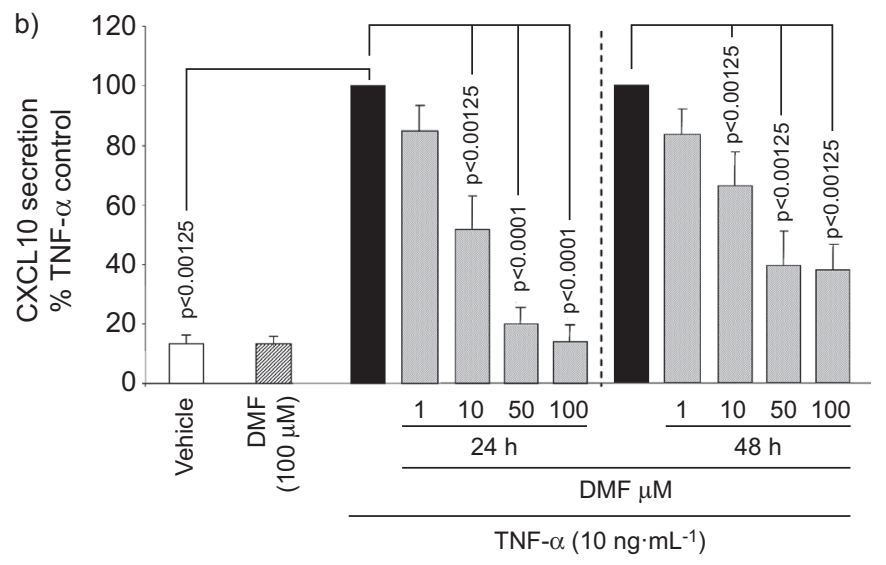

FIGURE 1. Dimethylfumarate (DMF) inhibits tumour necrosis factor (TNF)- $\alpha$ induced CXCL10 secretion and is supported by a glucocorticoid. a) Cells were stimulated with TNF- $\alpha\left(10 \mathrm{ng} \cdot \mathrm{mL}^{-1}\right)$ or were left untreated for 24 or $48 \mathrm{~h}$. CXCL10 was measured in the cell culture medium by ELISA. Data are presented as the mean \pm SEM of triplicate experiments in 10 independent cell lines and $p$-values were calculated by paired t-tests compared with nonstimulated cells. b) Airway smooth muscle cells (ASMCs) $(n=8)$ were pre-treated with DMF or the vehicle $(0.1 \%$ dimethylsulfoxide) for $1 \mathrm{~h}$ prior to stimulation with TNF- $\alpha$. CXCL10 secretion was measured by ELISA at 24 and $48 \mathrm{~h}$. Data are presented as a percentage of the CXCL10 production cells treated with TNF- $\alpha$ alone and $p$-values were calculated by one-way ANOVA. c) ASMCs ( $n=6)$ were treated with either fluticasone alone or in combination with DMF for $24 \mathrm{~h}$ before CXCL10 secretion was determined by ELISA. Data are presented as the mean \pm SEM of triplicate experiments in six independent ASMC lines, and p-values were calculated by ANOVA and corrected by the Bonferroni method. 
haemin $(1-10 \mu \mathrm{M})$ and cobalt protoporphyrin $(2-20 \mu \mathrm{M})$ were dissolved in dimethylsulfoxide (DMSO), and were diluted to the required concentration in serum-free medium. Glutathione ethylester (GSH-OEt) was dissolved to a final concentration of $1 \mathrm{mM}$ in serum-free medium. The cytotoxic efficacy of DMF concentrations was not significant, as reported previously [13].

\section{CXCL10 secretion by ASMCs}

ASMCs were grown to confluence and the growth medium was replaced with low-serum medium $(0.1 \%$ FBS) for $24 \mathrm{~h}$, before the DMF was added for $1 \mathrm{~h}$. The cells were then stimulated with TNF- $\alpha$ and/or IFN- $\gamma$ (both R\&D Systems, Minneapolis, MN, USA) and cell culture medium samples were collected 24 or $48 \mathrm{~h}$ later. CXCL10 and G-CSF protein levels were determined by ELISA following the manufacturer's instructions (ELISA Duo Set; R\&D Systems).

\section{Transfection with small interfering RNA}

Cells were seeded into 12-well culture plates $\left(1 \times 10^{5}\right.$ cells $\left.\cdot \mathrm{mL}^{-1}\right)$ and grown to $70 \%$ confluence in RPMI (10\% FBS). Cells were washed with FBS-free RPMI before being transiently transfected with $10 \mu \mathrm{M}$ small interfering RNA (siRNA), either targeting HO- 1 or the respective control siRNA, in $5 \mu \mathrm{L}$ siRNA transfection reagent (all Santa Cruz Biotechnology, Heidelberg,
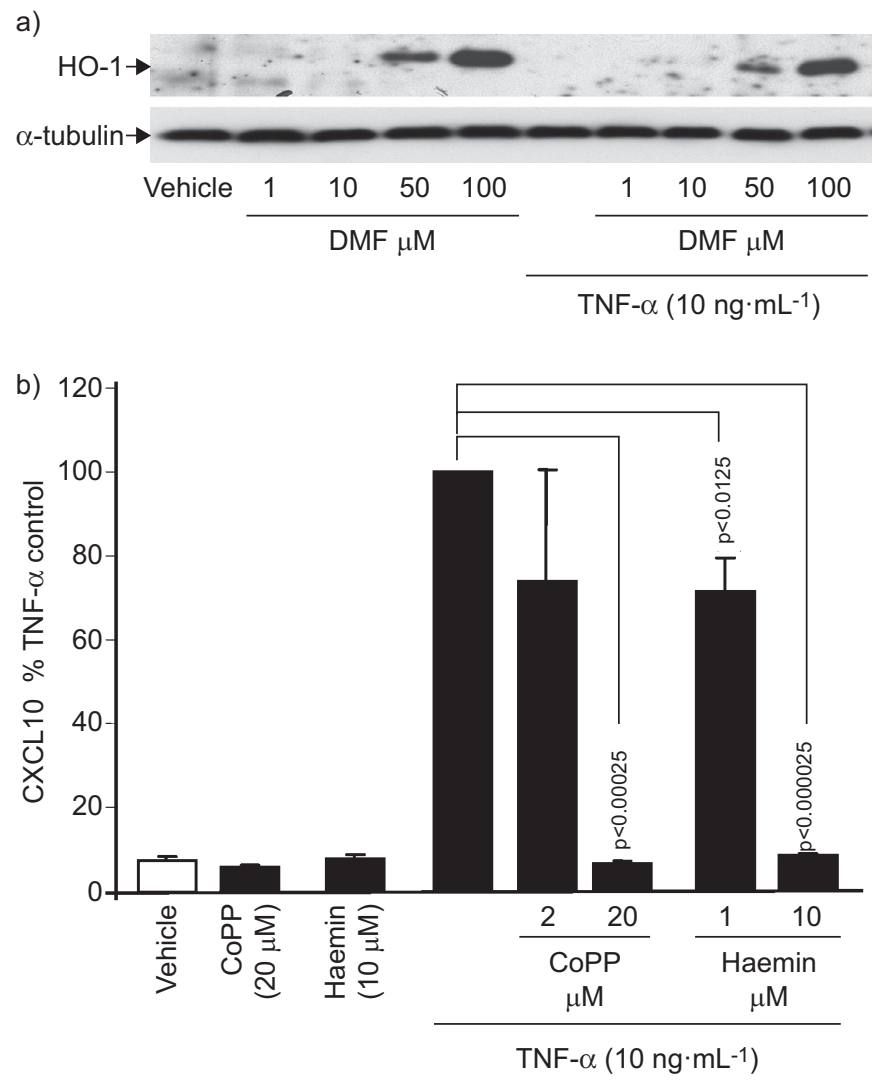

Germany). Cells incubated with transfection reagent alone served as negative controls. Six hours after siRNA treatment, the cells were stimulated with growth medium (RPMI, 10\% FBS and $1 \times$ antibiotics) for $20 \mathrm{~h}$. Cells were washed with PBS and incubated with DMF ( $50 \mu \mathrm{M})$ for 1 h before TNF- $\alpha$ or IFN- $\gamma(5$ or $10 \mathrm{ng} \cdot \mathrm{mL}^{-1}$ ) was added. After $24 \mathrm{~h}$, cell culture medium samples were collected and CXCL10 protein was quantified by ELISA.

\section{HO-1 expression and MAPK activation}

ASMCs were grown to confluence and were then serum starved for $24 \mathrm{~h}(0.1 \% \mathrm{FBS})$. The cells were pre-treated $(1 \mathrm{~h})$ with a single drug or a drug combination, before being stimulated with TNF- $\alpha$. Total cell lysate was collected at various time points $(0,5$, $10,15,30$ and $60 \mathrm{~min}$ ) to determine the expression and activation of MAPKs by immunoblotting. HO-1 expression was determined after $24 \mathrm{~h}$.

\section{Immunoblotting}

Protein extracts were size-fractionated by sodium dodecylsulfate-polyacrylamide gel electrophoresis and were transferred onto nitrocellulose membranes as described previously [13]. Protein transfer was confirmed by Ponceau staining. Membranes were incubated with blocking buffer $(5 \%(\mathrm{w} / \mathrm{v})$ skimmed dry milk in Tris-buffered saline containing $0.1 \%$

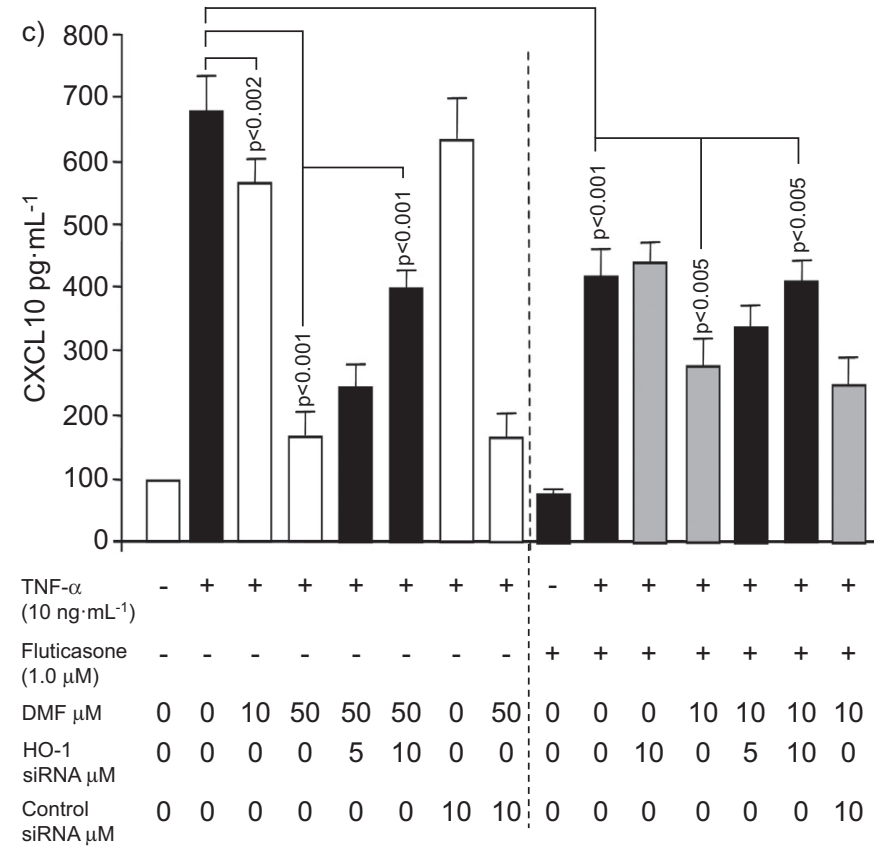

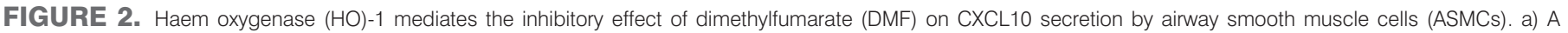

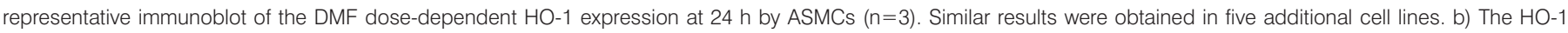

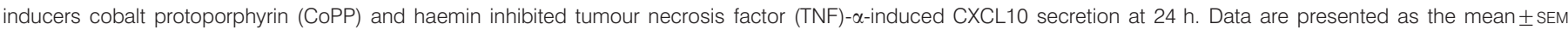

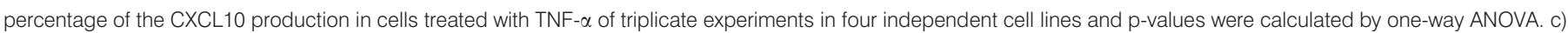

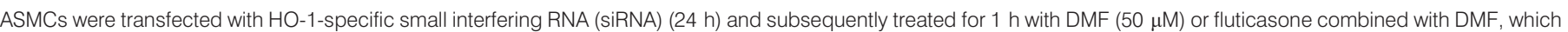

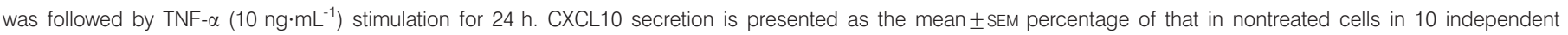
experiments, and p-values were calculated by ANOVA and corrected by the Bonferroni method. 


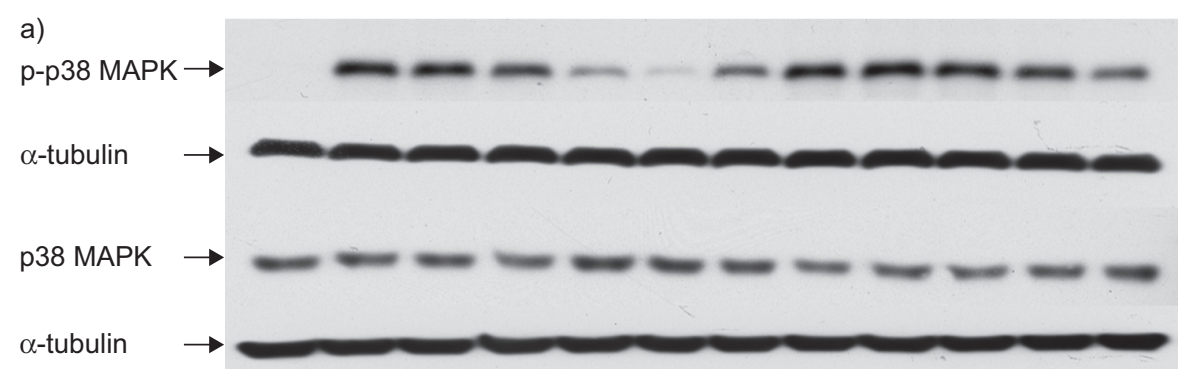

c)

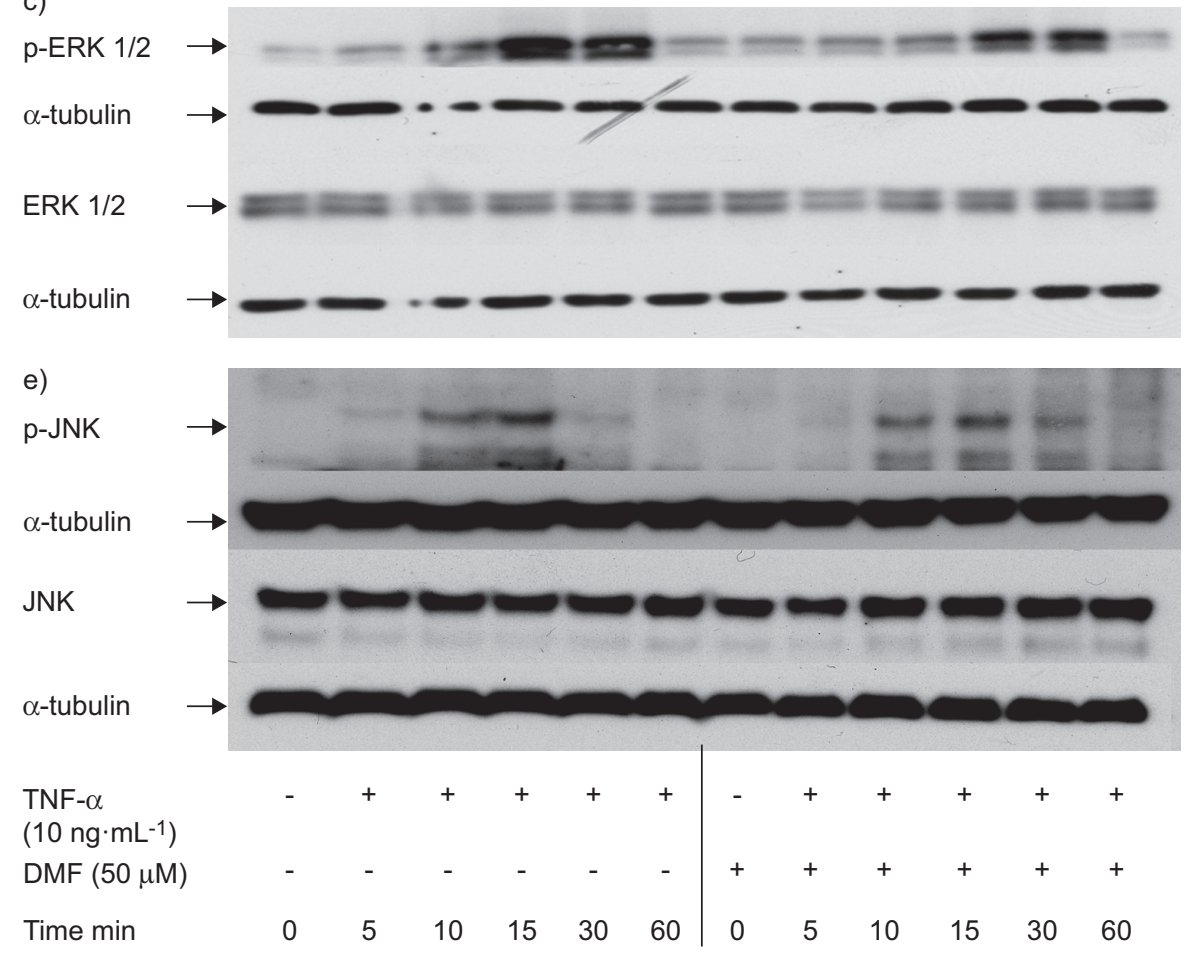

f)
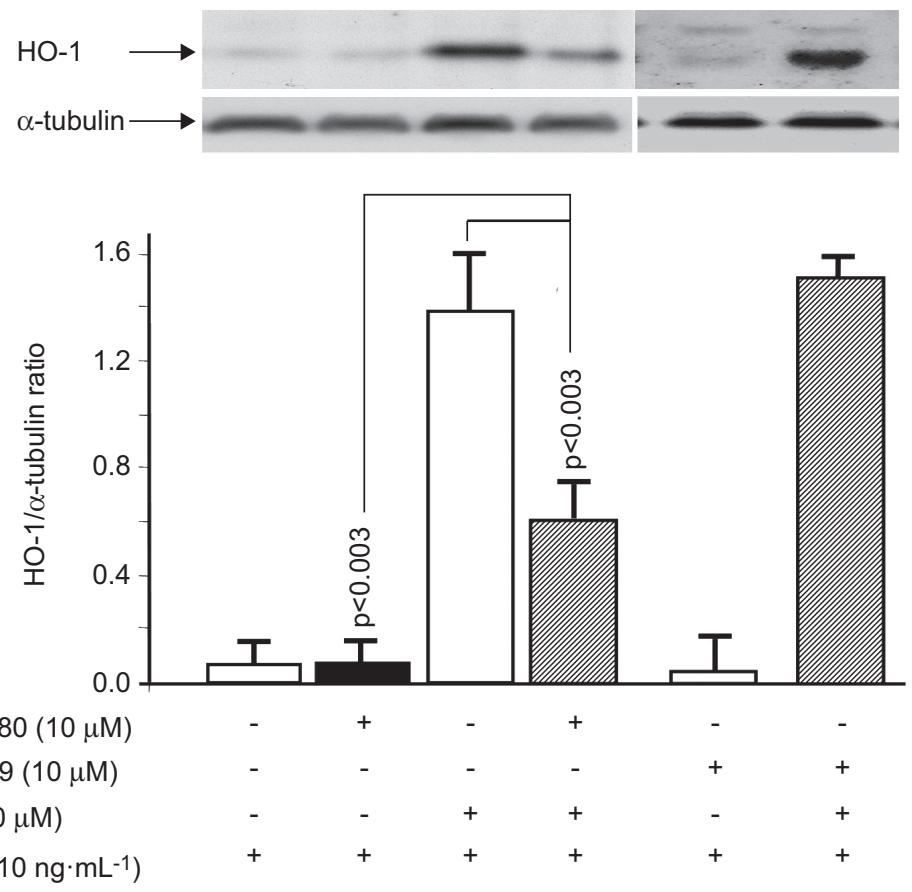

FIGURE 3. For figure legend see next page.
SB203580 $(10 \mu \mathrm{M})$ PD98059 $(10 \mu \mathrm{M})$ DMF $(50 \mu \mathrm{M})$ TNF- $\alpha\left(10 \mathrm{ng} \cdot \mathrm{mL}^{-1}\right)$
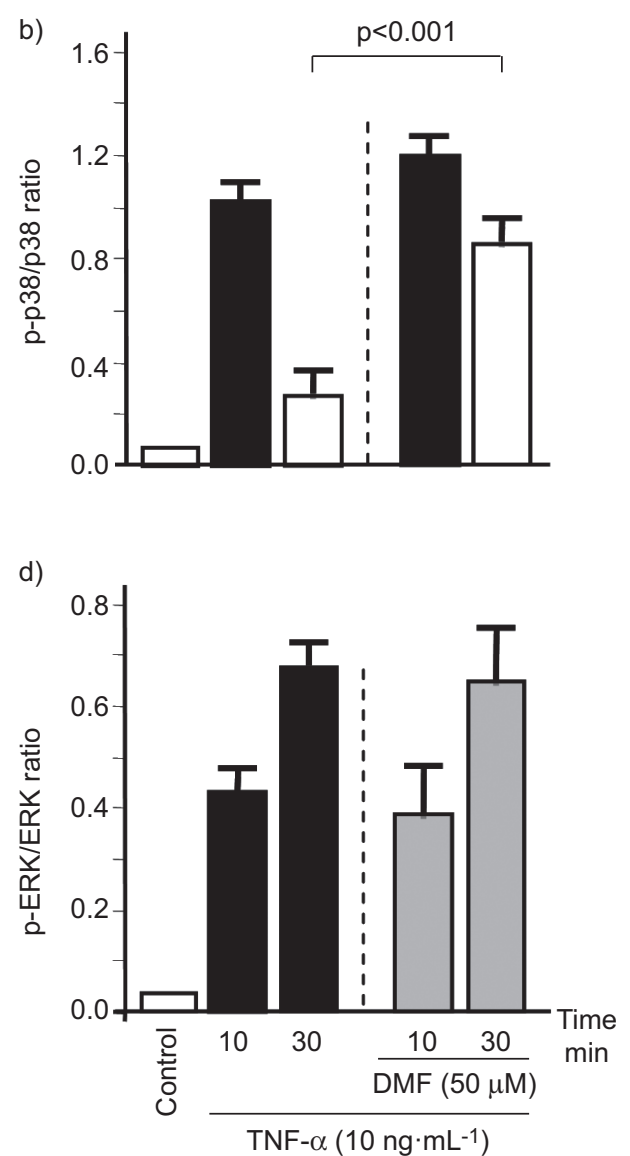
FIGURE 3. Dimethylfumarate (DMF) activates phosphorylation of p38 mitogen-activated protein kinase (MAPK), which mediates haem oxygenase (HO)-1 expression by airway smooth muscle cells. a) A representative immunoblot and b) quantification of the kinetics of the phosphorylation of p38 MAPK (p-p38 MAPK) by tumour necrosis factor (TNF)- $\alpha$ in the presence and absence of DMF $(n=3)$. c) A representative immunoblot and d) quantification of the kinetics of extracellular signal-regulated kinase (ERK) $1 / 2$ phosphorylation (p-ERK1/2) by TNF- $\alpha$ and its enhancement by DMF $(n=3)$. e) A representative immunoblot of the kinetics of c-Jun N-terminal kinase (JNK) MAPK phosphorylation ( $p$-JNK) by TNF- $\alpha$ and in the presence and absence of DMF ( $n=3)$. f) A representative immunoblot of DMF-induced HO-1 expression and its reduction by the P38 MAPK inhibitor SB203580 in the presence of TNF- $\alpha(n=3)$. Similar results were obtained in three additional cell lines and graphs represent the mean \pm SEM of all experiments. p-values were calculated by one-way ANOVA and corrected by the Bonferroni method.

Tween 20) for $1 \mathrm{~h}$ at room temperature and were then incubated with a primary antibody specific to either p38, phosphorylated p38, extracellular signal-regulated kinase (ERK)1/2, phosphorylated ERK1/2, c-Jun N-terminal kinase (JNK), phosphorylated JNK (all Cell Signaling Technology, Beverly, MA, USA), HO-1 (Calbiochem, Luzern, Switzerland) or $\alpha$-tubulin (Santa Cruz Biotech). Binding of primary antibodies was detected by horseradish peroxidase-conjugated immunoglobulin (Ig)G antibodies diluted 1:2,000-1:40,000 (anti-rabbit or anti-mouse IgG; Santa Cruz Biotech). Protein bands were visualised by enhanced chemiluminescence (Pierce Biotechnology, Rockford, IL, USA).

\section{Data analysis}

Triplicate or duplicate results of each experiment were averaged for each primary ASMC line and from these data, the mean \pm SEM was calculated. The statistical analysis was performed using oneway ANOVA. p-values were corrected by the Bonferroni method and are presented in the respective figures.

\section{RESULTS}

DMF inhibits TNF- $\alpha$-induced CXCL10 secretion by ASMCs

ASMC were stimulated with TNF- $\alpha\left(10 \mathrm{ng} \cdot \mathrm{mL}^{-1}\right)$ or were left unstimulated for 24 and $48 \mathrm{~h}$ before CXCL10 levels were determined in the cell culture medium. As shown in figure 1a, unstimulated ASMCs released low levels of CXCL10 (mean \pm SEM $69.9 \pm 27.1 \mathrm{pg} \cdot \mathrm{mL}^{-1}$ ) within $24 \mathrm{~h}$, while stimulation with TNF- $\alpha$ significantly upregulated CXCL10 secretion at $24 \mathrm{~h}(1,044 \pm 284$ $\left.\mathrm{pg} \cdot \mathrm{mL}^{-1}\right)$ and $48 \mathrm{~h}\left(1,382 \pm 316 \mathrm{pg} \cdot \mathrm{mL}^{-1}\right)$. Pre-incubation with DMF $(1-100 \mu \mathrm{M})$ dose-dependently reduced the TNF- $\alpha$-induced CXCL10 secretion to a similar extent, while DMF alone had no effect on unstimulated ASMCs (fig. 1b). As expected, fluticasone (0.1-10 $\mu \mathrm{M})$ inhibited TNF- $\alpha$-induced CXCL10 secretion and in combination with DMF $(10 \mu \mathrm{M})$, this effect was stronger compared with each drug alone (fig. 1c).

\section{DMF inhibits CXCL10 secretion by ASMCs through HO-1}

$\mathrm{HO}-1$ expression was determined by immunoblotting. DMF induced HO-1 expression dose dependently, while TNF- $\alpha$ neither induced nor altered DMF-induced HO-1 expression (fig. 2a). In order to corroborate this inhibitory effect of HO-1 on CXCL10 secretion, ASMCs were pre-incubated for $1 \mathrm{~h}$ with haemin $(1-10 \mu \mathrm{M})$ or cobalt protoporphyrin $(2-20 \mu \mathrm{M})$ before stimulation with TNF- $\alpha$. Both HO-1 inducers dose-dependently reduced TNF- $\alpha$-induced CXCL10 secretion by ASMCs but had no effect on unstimulated cells (fig. 2b).

The role of HO-1 in DMF's inhibitory effect on TNF- $\alpha$-induced CXCL10 secretion was also studied in ASMCs that had been transiently transfected with HO-1 siRNA prior to DMF and TNF- $\alpha$ treatment. As shown in figure $2 c$, the inhibitory effect of DMF $(50 \mu \mathrm{M})$ on CXCL10 secretion was dose-dependently counteracted by HO-1 siRNA $(p<0.05)$, while control siRNA had no such effect. Importantly, the inhibitory effect of fluticasone alone on TNF- $\alpha$-induced CXCL10 was not affected by the presence of HO-1 siRNA, which, when treated with the combined drugs, counteracted the effect of DMF (fig. 2c).

\section{DMF increases and prolongs TNF- $\alpha$-induced p38 MAPK phosphorylation}

TNF- $\alpha\left(10 \mathrm{ng} \cdot \mathrm{mL}^{-1}\right)$ strongly induced p38 MAPK phosphorylation within $5 \mathrm{~min}$, which declined afterwards and reached baseline level at $60 \mathrm{~min}$ (fig. $3 \mathrm{a}$ and b). DMF $(50 \mu \mathrm{M})$ alone activated p38 MAPK phosphorylation and, when combined with TNF- $\alpha$, it increased and prolonged p38 MAPK phosphorylation (fig. 3a and b). Total p38 MAPK expression was not affected by any treatment (fig. 3a and b). TNF- $\alpha$ induced ERK1/2 MAPK and JNK phosphorylation, neither of which was affected by DMF (fig. 3c-e). No change was observed for total ERK1/2 MAPK or total JNK levels with any treatment (fig. 3c-e).

To link DMF-induced p38 MAPK phosphorylation and HO-1 expression, ASMCs were treated with DMF and either the p38 MAPK inhibitor SB203580 or the ERK1/2 inhibitor PD98059. Inhibition of p38 MAPK prevented the expression of HO-1 by DMF, whereas SB203580 alone or PD98059 had no significant effect (fig. 3f).

\section{The effect of DMF on p38 MAPK, HO-1 and CXCL10 depends on intracellular GSH}

To understand the role of intracellular GSH on DMF's effects, ASMCs were pre-treated with GSH-OEt before the addition of any other treatment. GSH-OEt completely inhibited DMFinduced p38 MAPK phosphorylation and it prevented the additive effect of DMF on TNF- $\alpha$-induced p38 MAPK phosphorylation but had no effect on TNF- $\alpha$ alone (fig. $4 a$ ). Increasing the intracellular GSH concentration prevented DMF-induced HO-1 expression both in the absence and presence of TNF- $\alpha$ (fig. 4b). As shown in figure 4c, GSH-OEt treatment significantly reduced the inhibitory effect of DMF on TNF- $\alpha$-induced CXCL10 secretion.

\section{DMF inhibits TNF- $\alpha-$ and IFN- $\gamma$-induced CXCL10 secretion via $\mathrm{HO}-1$}

INF- $\gamma$ induced CXCL10 secretion and this was inhibited by DMF (fig. 4d). Combined TNF- $\alpha$ and IFN- $\gamma$ showed an additive stimulatory effect on CXCL10 secretion, which was also prevented by DMF pre-incubation (fig. $4 \mathrm{~d}$ ). When ASMCs were pre-treated with HO-1 siRNA, the inhibitory effect of DMF on TNF- $\alpha$ - and IFN- $\gamma$-induced CXCL10 secretion was completely reversed (fig. $4 \mathrm{~d})$. Control siRNA $(10 \mu \mathrm{M})$ did not alter TNF- $\alpha / \mathrm{IFN}-\gamma-$ induced CXCL10 secretion or the inhibitory effect of DMF (fig. 4d).

DMF dose-dependently inhibited IFN- $\gamma$-induced CXCL10 secretion as well as the stimulatory effect of the combined stimuli (fig. 5a). Supplementation of glutathione prevented the inhibitory effect of DMF in ASMCs stimulated with either IFN- $\gamma$ alone or with the combined stimuli (fig. 5a). 
a)

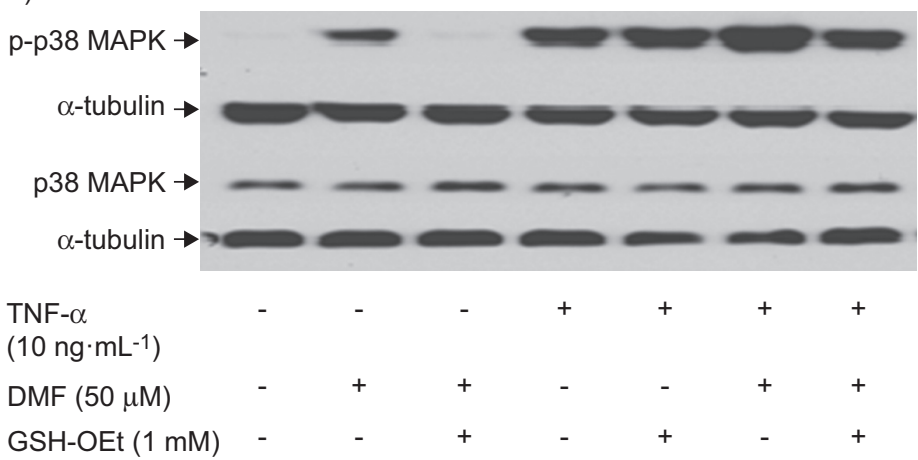

b)

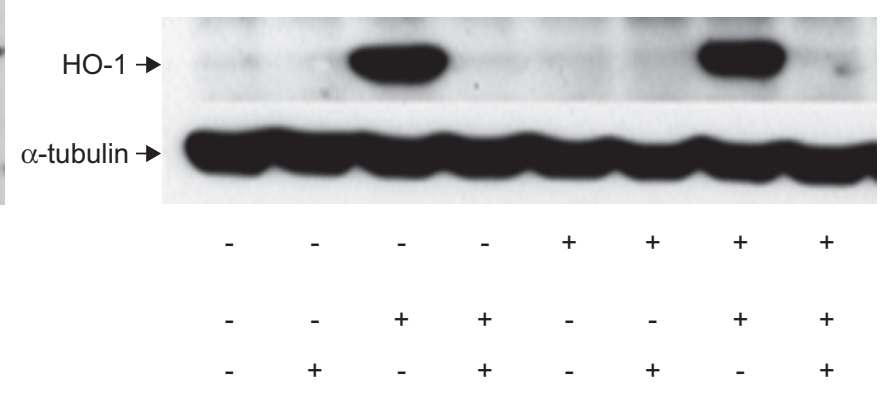

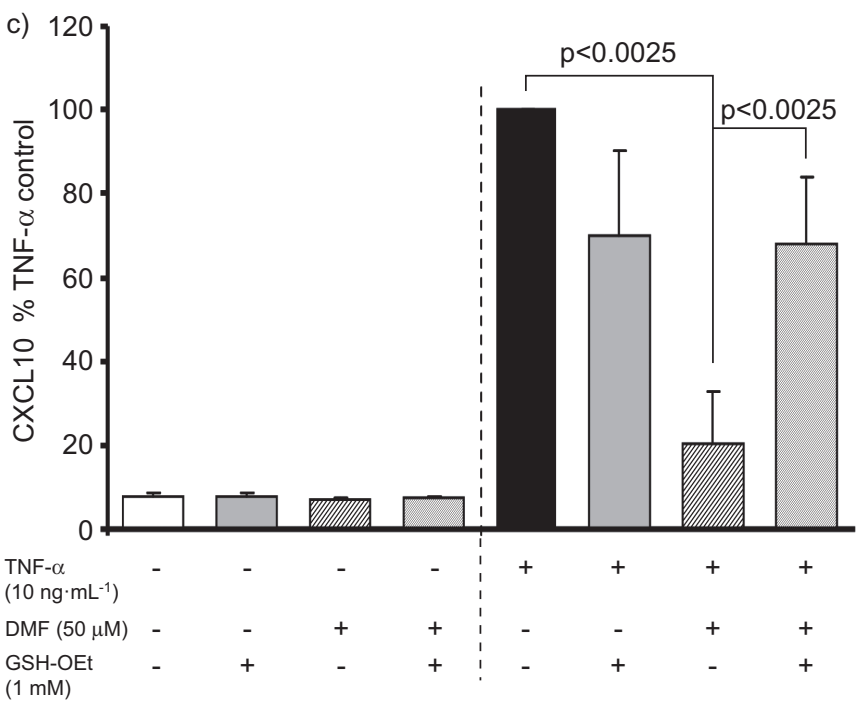

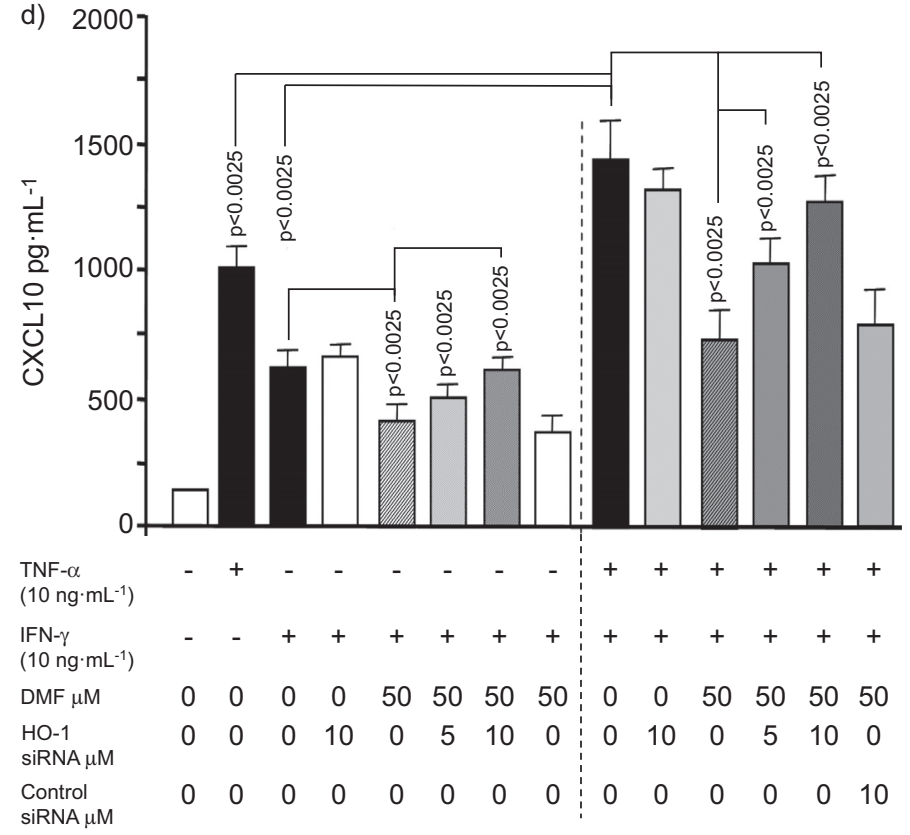

FIGURE 4. Glutathione reverses the effects of dimethylfumarate (DMF) on p38 mitogen-activated protein kinase (MAPK) phosphorylation, haem oxygenase (HO)-1 expression and CXCL10 secretion in airway smooth muscle cells (ASMCs). a) Representative immunoblot $(n=4)$ of the reversing effect of glutathione ethylester (GSH-OEt) on DMF- and tumour necrosis factor (TNF)- $\alpha$-induced phosphorylation of p38 MAPK in ASMCs stimulated for 30 min. b, c) The reversing effect of GSH-OEt on DMF-dependent HO-1 expression and inhibition of TNF- $\alpha$-induced CXCL10 secretion. Data are presented as the mean \pm SEM of triplicate experiments in four independent cell lines, and pvalues were calculated by ANOVA and corrected by the Bonferroni method. d) HO-1 small interfering RNA (siRNA) counteracts the inhibitory effect of DMF on TNF- $\alpha$ - and IFN- $\gamma$-induced CXCL10 secretion. Data are presented as the mean \pm SEM of triplicate experiments in six ASMC lines, and p-values were calculated by ANOVA and corrected by the Bonferroni method.

TNF- $\alpha$ and IFN- $\gamma$, alone or in combination, induced the secretion of G-CSF (fig. 5b). DMF alone had no effect on G-CSF secretion but partially reduced G-CSF secretion induced by TNF- $\alpha$, while it had no effect on IFN- $\gamma$-dependent G-CSF secretion (fig. 5b). In ASMCs stimulated with both TNF- $\alpha$ and IFN- $\gamma$, DMF treatment partly reduced G-CSF secretion (fig. 5b). Supplementation of GSH-OEt compensated the inhibitory effect of DMF on TNF- $\alpha$ but not on IFN- $\gamma$-induced G-CSF secretion (fig. 5b).

\section{DISCUSSION}

In this study, we provide evidence that DMF has a significant immunomodulatory effect on cultured human primary ASMCs. DMF inhibited TNF- $\alpha$-induced CXCL10 and, importantly, showed a steroid-sparing effect when combined with fluticasone. The inhibitory effect of DMF was mediated via the induction and sustained phosphorylation of p38 MAPK, which subsequently induced HO-1 expression. In addition, IFN- $\gamma$-induced CXCL10 secretion, which was reported to be less sensitive to steroids in ASMCs, was also inhibited by DMF. Increasing the intracellular level of GSH prior to DMF treatment reversed all the drug's effects. Thus, our data indicate that DMF has potential for the treatment of chronic inflammatory lung diseases.

The pathological increase of ASMC number and size in asthmatic airways was first reported by HubER and KESSLER [15] in 1926 and this finding was later confirmed to occur in childhood asthma, often before any sign of inflammation is detected [16, 17]. In asthma, ASMCs constrict and narrow the airways upon exposure to a wide range of triggers, thereby limiting airflow. Furthermore, ASMCs contribute to local inflammation by secreting a large range of pro-inflammatory cytokines [3]. Based on these observations, it is plausible that 

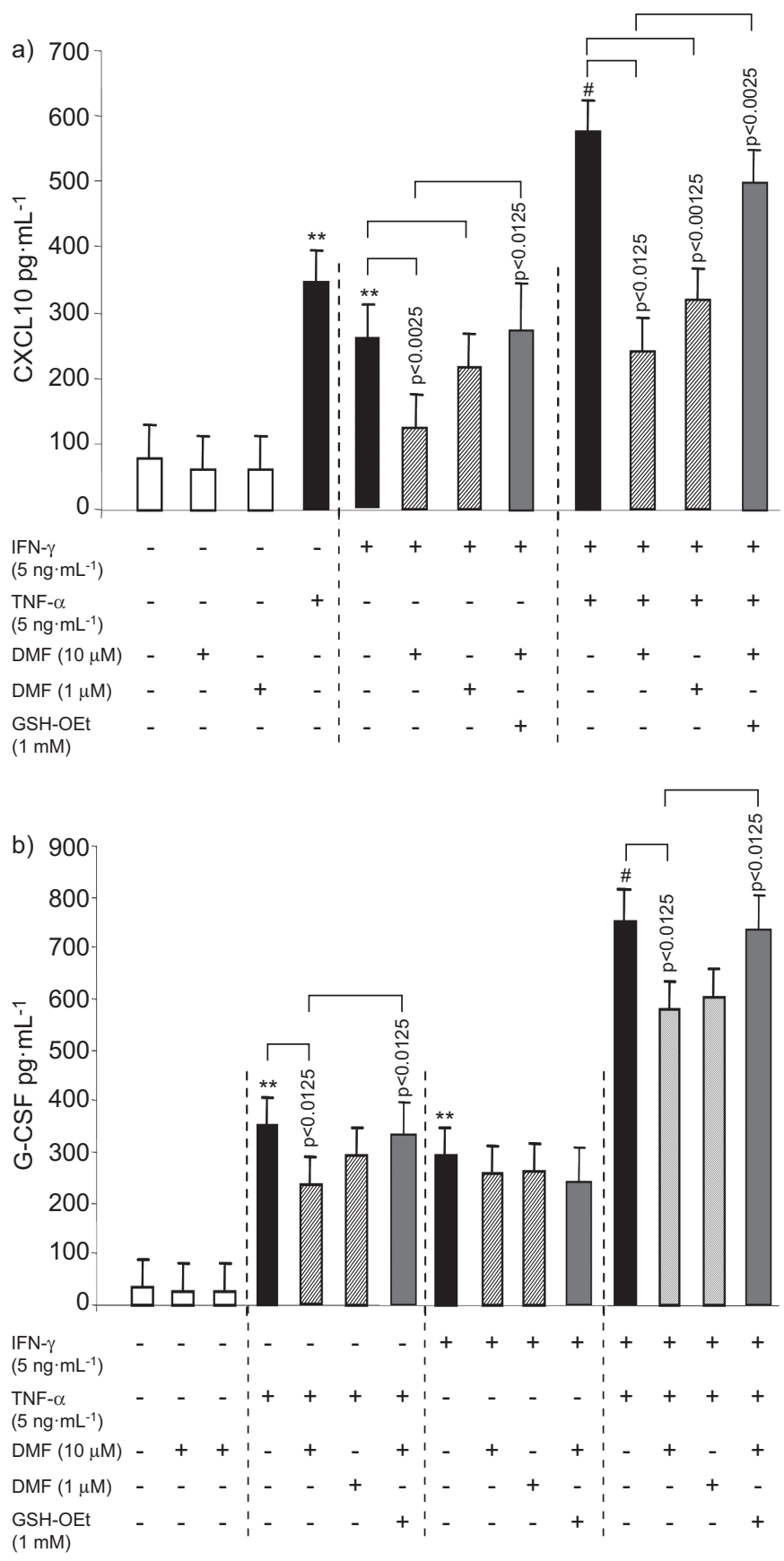

FIGURE 5. The inhibition of CXCL10 and granulocyte colony-stimulating factor (G-CSF) secretion through dimethylfumarate (DMF) and its reversal by glutathione is stimulus- and cytokine-specific. a) Six airway smooth muscle cell (ASMC) lines were treated with either tumour necrosis factor (TNF)- $\alpha$ or interferon (IFN)- $\gamma$ alone or both combined. Pre-treatment with DMF dose-dependently reduced CXCL10 secretion, which was counteracted by glutathione supplementation. The p-values were calculated by ANOVA and corrected by the Bonferroni method. b) The effect of DMF and glutathione supplementation on G-CSF secretion by TNF- $\alpha$ and/or IFN- $\gamma$ stimulation at $24 \mathrm{~h}$. Data are presented as the mean \pm SEM of triplicate experiments in four independent cell lines. The $p$-values were calculated by ANOVA and corrected by the Bonferroni method. GSH-OEt: glutathione ethylester. ${ }^{* *}: p<0.01$ compared with unstimulated ASMCs; ${ }^{*}: p<0.01$ compared with a single stimulus. targeting ASMC activity and cytokine secretion may help to resolve inflammation in chronic lung diseases.

Our observation that DMF down-regulated the secretion of CXCL10 by ASMCs is of particular importance as CXCL10 is one of the strongest recruitment factors for mast cells into the inflamed airway tissue [4-6]. In this study, we showed that DMF inhibited IFN- $\gamma$-induced CXCL10 through HO-1 expression. This observation strengthens the argument for the therapeutic use of DMF in asthma therapy as IFN- $\gamma$-induced CXCL10 secretion was insensitive to steroid treatment [9]. Furthermore, in TNF- $\alpha$ activated ASMCs the reductive effect of the combination of DMF with fluticasone was stronger than that of each drug alone.

In human lung fibroblasts and ASMCs, we have shown that DMF inhibited PDGF-BB and TNF- $\alpha$-induced the secretion of IL-6 $[18,19]$. This effect was mediated by the inhibition of two transcription factors, nuclear factor (NF)- $\mathrm{kB}$ p65 and activator protein-1, by DMF in fibroblasts $[18,19]$. We further provided evidence that DMF reduces histone 3 and cAMP response element-binding protein (CREB) phosphorylation [18, 19]. The inhibitory effect of DMF on NF- $\mathrm{KB}$ and CREB may explain its effect on IFN- $\gamma$-induced CXCL10 secretion, as both transcription factors mediate INF- $\gamma$ signalling to CXCL10 activity $[9,20]$. These findings suggested a more general mode of action of DMF by reducing the binding of several transcription factors that stimulate gene expression.

Previously, we reported that DMF significantly reduces the PDGF-BB-induced proliferation of ASMCs through induction of HO-1, suggesting that DMF may reduce airway remodelling [13]. In this study, we were interested in whether DMF-mediated up-regulation of HO-1 is also involved in DMF's anti-inflammatory effects. A beneficial anti-inflammatory effect of HO-1 has been suggested in other allergic diseases and may indicate an overall protective anti-inflammatory effect of DMF [21, 22]. Similar to our results, pyrrolidine dithiocarbamate induced HO-1 expression, which reduced eosinophil and T-cell counts in bronchoalveolar lavage fluid, as well as airway hyperresponsiveness in a mouse model of allergic airway disease [23]. The observed reduced eosinophil activity may be related to our finding that DMF reduced the secretion of eotaxin and RANTES by blocking histone 3 phosphorylation [19]. Further beneficial effects of increasing HO-1 expression in the inflamed lung may be related to reduced airway inflammation, mucus secretion, oxidative stress and airway hyper-responsiveness, which were shown in an asthma model of ovalbumin-sensitised guinea pigs [22]. In our study, DMF induced HO-1 in the presence of TNF- $\alpha$ and this effect was abrogated in ASMCs that had been treated with HO-1 siRNA. Furthermore, two additional HO-1 inducers, haemin and cobalt protoporphyrin, also reduced TNF- $\alpha$-induced CXCL10 secretion, thus supporting the hypothesis that HO-1 mediates the anti-inflammatory effect of DMF.

Based on our earlier studies, we showed that DMF enhanced TNF- $\alpha$-induced HO-1 expression through p38 MAPK phosphorylation. Furthermore, GSH supplementation compensated the effects of DMF on p38 MAPK phosphorylation and CXCL10 secretion. Our findings are in line with another study where GSH depletion increased the phosphorylation of p38 MAPK in C6 glioma cells [24]. Furthermore, DMF depleted GSH levels, and thereby inhibited IL-12 and IFN- $\gamma$ secretion by peripheral blood 
mononuclear cells [10]. In human lung fibroblasts, the reduction of intracellular GSH induced HO-1 [25]. Furthermore, in pancreatic stellate cells, the activation of p38 MAPK augmented HO-1 expression [26]. In regard to CXCL10 secretion, HO-1 reduced CXCL10 expression by isolated macrophage cells [27].

To further characterise the signalling-specific action of DMF, we stimulated cells with IFN- $\gamma$, a known inducer of CXCL10 [9]. We confirmed that IFN- $\gamma$ combined with TNF- $\alpha$ has an additive stimulatory effect on CXCL10 secretion and on G-CSF secretion $[9,28]$. These studies suggested that NF- $\kappa B$ activation mediates TNF- $\alpha$-induced CXCL10 secretion but is only marginally involved when cells are stimulated by IFN- $\gamma$. Our data suggest that DMF controls TNF- $\alpha$ - and IFN- $\gamma$-induced CXCL10 secretion by human ASMCs by lowering intracellular GSH levels.

In conclusion, we describe here a new molecular signalling mechanism by which DMF inhibits cytokine-induced CXCL10 secretion by ASMCs. In addition, we show that DMF has steroid-saving properties. Therefore, DMF can be considered as a modulator of the immune response in chronic inflammatory airway diseases.

\section{SUPPORT STATEMENT}

The study was supported by an unrestricted research grant of the Swiss National Foundation (grant 310030_130740/1) and by an asthma research grant to M. Roth in 2010 from the Gottfried and Julia Bangerter Ryhner-Stiftung, Basel, Switzerland.

\section{STATEMENT OF INTEREST}

None declared.

\section{ACKNOWLEDGEMENTS}

We thank C.T. S'ng (University Hospital Basel, Basel, Switzerland) for his help preparing this manuscript.

\section{REFERENCES}

1 Holgate ST. Pathogenesis of asthma. Clin Exp Allergy 2008; 38: 872-897.

2 Holgate ST, Roberts G, Arshad HS, et al. The role of the airway epithelium and its interaction with environmental factors in asthma pathogenesis. Proc Am Thorac Soc 2009; 6: 655-659.

3 Damera G, Tliba O, Panettieri RA Jr. Airway smooth muscle as an immunomodulatory cell. Pulm Pharmacol Ther 2008; 22: 353-359.

4 Siddiqui S, Hollins F, Saha S, et al. Inflammatory cell microlocalisation and airway dysfunction: cause and effect? Eur Respir J 2007; 30: 1043-1056.

5 Brightling CE, Bradding P, Symon FA, et al. Mast-cell infiltration of airway smooth muscle in asthma. $N$ Engl J Med 2002; 346: 1699-1705.

6 Brightling CE, Ammit AJ, Kaur D, et al. The CXCL10/CXCR3 axis mediates human lung mast cell migration to asthmatic airway smooth muscle. Am J Respir Crit Care Med 2005; 171: 1103-1108.

7 McCormack PL, Lyseng-Williamson KA. Budesonide/formoterol - a review of its use as maintenance and reliever inhalation therapy in asthma. Drugs 2007; 67: 2407-2431.

8 Adcock IM, Caramori G, Chung KF. New targets for drug development in asthma. Lancet 2008; 372: 1073-1087.

9 Clarke DL, Clifford RL, Jindarat S, et al. TNF $\alpha$ and IFN $\gamma$ synergistically enhance transcriptional activation of CXCL10 in human airway smooth muscle cells via STAT-1, NF-kB, and the transcriptional coactivator CREB-binding protein. J Biol Chem 2010; 285: 29101-29110.
10 Lehmann JC, Listopad JJ, Rentzsch CU, et al. Dimethylfumarate induces immunosuppression via glutathione depletion and subsequent induction of heme oxygenase 1. J Invest Dermatol 2007; 127: 835-845.

11 Nelson KC, Carlson JL, Newman ML, et al. Effect of dietary inducer dimethylfumarate on glutathione in cultured human retinal pigment epithelial cells. Invest Ophthalmol Vis Sci 1999; 40: 1927-1935.

12 Seidel P, Merfort I, Hughes JM, et al. Dimethylfumarate inhibits NF- $\mathrm{BB}$ function at multiple levels to limit airway smooth muscle cell cytokine secretion. Am J Physiol Lung Cell Mol Physiol 2009; 297: L326-L339.

13 Seidel P, Goulet S, Hostettler K, et al. DMF inhibits PDGF-BB induced airway smooth muscle cell proliferation through induction of heme-oxygenase-1. Respir Res 2010; 11: 145.

14 Hoefnagel JJ, Thio HB, Willemze R, et al. Long-term safety aspects of systemic therapy with fumaric acid esters in severe psoriasis. $\mathrm{Br}$ J Dermatol 2003; 149: 363-369.

15 Huber H, Kessler K. The pathology of bronchial asthma. Arch Intern Med 1922; 30: 689-760.

16 Jesudason EC. Airway smooth muscle: an architect of the lung? Thorax 2009; 64: 541-545.

17 Jenkins HA, Cool C, Szefler SJ, et al. Histopathology of severe childhood asthma: a case series. Chest 2003; 124: 32-41.

18 Seidel P, Merfort I, Tamm M, et al. Inhibition of NF- $\mathrm{kB}$ and AP-1 by dimethylfumarate correlates with down-regulated IL-6 secretion and proliferation in human lung fibroblasts. Swiss Med Wkly 2010; 140: w13132.

19 Seidel $\mathrm{P}$, Roth $\mathrm{M}, \mathrm{Ge} \mathrm{Q}$, et al. IKB- $\alpha$ glutathionylation and reduced histone $\mathrm{H} 3$ phosphorylation inhibit eotaxin and RANTES. Eur Respir J 2011; 38: 1444-1452.

20 Hardaker EL, Bacon AM, Carlson K, et al. Regulation of TNF- $\alpha$ - and IFN- $\gamma$-induced CXCL10 expression: participation of the airway smooth muscle in the pulmonary inflammatory response in chronic obstructive pulmonary disease. FASEB J 2004; 18: 191-193.

21 Pae HO, Lee YC, Chung HT. Heme oxygenase-1 and carbon monoxide. emerging therapeutic targets in inflammation and allergy. Recent Pat Inflamm Allergy Drug Discov 2008; 2: 159-165.

22 Almolki A, Taillé C, Martin GF, et al. Heme oxygenase attenuates allergen-induced airway inflammation and hyperreactivity in guinea pigs. Am J Physiol Lung Cell Mol Physiol 2004; 287: L26-L34.

23 Kwak HJ, Song JS, Heo JY, et al. Protective effects of pyrrolidine dithiocarbamate against airway inflammation in the ovalbumininduced mouse model. Eur J Pharmacol 2008; 590: 355-362.

24 Kim SM, Park JG, Baek WK, et al. Cadmium specifically induces MKP-1 expression via the glutathione depletion-mediated p38 MAPK activation in C6 glioma cells. Neurosci Lett 2008; 440: 289-293.

25 Baglole CJ, Sime PJ, Phipps RP. Cigarette smoke-induced expression of heme oxygenase- 1 in human lung fibroblasts is regulated by intracellular glutathione. Am J Physiol Lung Cell Mol Physiol 2008; 295: L624-L636.

26 Schwer CI, Mutschler M, Stoll P, et al. Carbon monoxide releasing molecule-2 inhibits pancreatic stellate cell proliferation by activating p38 mitogen-activated protein kinase/heme oxygenase-1 signaling. Mol Pharmacol 2010; 77: 660-669.

27 Tsuchihashi S, Zhai Y, Bo Q, et al. Heme oxygenase-1 mediated cytoprotection against liver ischemia and reperfusion injury: inhibition of type-1 interferon signaling. Transplantation 2007; 83: $1628-1634$.

28 Taillé C, Almolki A, Benhamed M, et al. Heme oxygenase inhibits human airway smooth muscle proliferation via a bilirubindependent modulation of ERK1/2 phosphorylation. J Biol Chem 2003; 278: 27160-27168. 